\title{
Role of Dimensionless Parameters in Modelling MEMS Hot-arm Actuators
}

\author{
Thomas M. Adams', Jason T. Collins², Johann Fernando ${ }^{3}$, Sarah M. Griffin ${ }^{4}$, Robert L. Kreft ${ }^{5}$, \\ Helio Kuok $^{6}$, Alexander E. McFarland ${ }^{7}$, Ethan J. Wasylewski ${ }^{8}$ \\ ${ }^{1}$ Rose-Hulman Institute of Technology \\ 5500 Wabash Avenue, Terre Haute, IN, USA \\ adams1@rose-hulman.edu \\ ${ }^{2}$ Canterbury School \\ 3210 Smith Road, Fort Wayne, IN 46804 \\ jcollins@canterburyschool.org \\ ${ }^{3}$ Eastside Catholic School \\ 232 228th Ave SE, Sammamish, WA, USA \\ Johann.Fernando@EastsideCatholicSchool.org \\ ${ }^{4}$ Northern Nevada Math Club \\ 7231 Pilot Court, Sparks, NV, USA \\ SarahMGriffin0@gmail.com \\ ${ }^{5}$ Roanoke Valley Governor's School \\ 2104 Grandin Road SW, Roanoke, VA, USA \\ rlkreft911@aol.com \\ ${ }^{6}$ Sha Tin College \\ No. 3 Lai Wo Lane Fo Tan, Sha Tin Hong Kong \\ helio.kuok@gmail.com \\ ${ }^{7}$ Highlands Latin School \\ 10901 Shelbyville Road, Louisville, Kentucky \\ mcfarland.alexander2000@gmail.com \\ ${ }^{8}$ Detroit Catholic Central High School \\ 27225 Wixom Road, Novi, MI, USA \\ 18wasylewskie@catholiccentral.net
}

\begin{abstract}
The current work presents a novel approach to analysing traditional U-shaped MEMS hot-arm actuators via the use of dimensional analysis. Two dimensionless groups, a thermo-electro parameter and a geometric parameter, are proposed in order to describe actuator behaviour, and the interpretation of each is discussed. Deflection data for six different actuators collected from five different studies are correlated using the dimensionless groups, resulting in a semi-empirical relationship that offers good predictability. In general, actuator deflection shows a strong linear dependence on the thermo-electro parameter, with large values of the geometric parameter resulting in smaller deflections.
\end{abstract}

Keywords: Hot-Arm Actuators, MEMS, MEMS Actuation, MEMS Transducers, Dimensional Analysis.

\section{Introduction}

Hot-arm actuators are among the most popular micro-electro-mechanical systems (MEMS) devices. Also known as Comtois actuators or simply heatuators, hot-arm actuators operate at lower voltages than electrostatic actuators, and often exhibit better force-displacement characteristics as well. The principle of operation is that of thermal expansion via Joule heating, which induces a mechanical stress in the device. This stress in turn bends the MEMS structure, and thus produces 
the actuation [1]. Actuation of optical devices is a common application of hot-arm actuators, as is their use in micromotors [2]-[3].

The operating principle of hot-arm actuation is depicted in Fig. 1. A voltage applied to the contact pads creates an electric current flowing through the hot arm, cold arm, and flexure, respectively. The long thin hot-arm has a much higher electrical resistance than the thicker cold arm, resulting in a large temperature difference between the two during operation. Owing to its larger thermal expansion, a compressive stress is induced in the hot-arm, which bends the entire structure downward, effecting actuation [4]. Total lengths of such devices range from $180 \mu \mathrm{m}$ to $1.5 \mathrm{~mm}$.

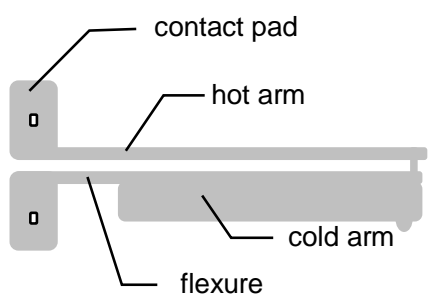

(a)

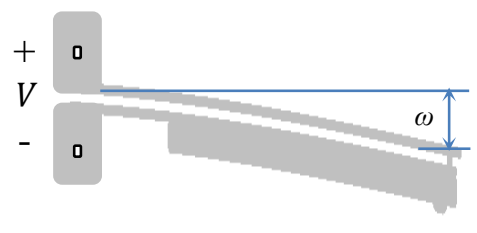

(b)

Fig. 1: Illustration of hot-arm actuation: (a) Actuator before voltage is applied. (b) Joule heating and thermal expansion cause the actuator to bend.

\section{Previous Work}

Many researchers have investigated hot-arm actuation, producing models that vary widely in approach and complexity ([4-15]). One of the most significant obstacles in such modelling is that the thermal, electrical, and mechanical domains are coupled with one another. As such, the use of finite element analysis (FEA) software packages is common ([10] and [15]), as are highly detailed analytical models, which generally solve for spatial distributions of temperature and stress en route to calculating tip deflection ([8]-[9]). The advantages of these approaches include their high predictive accuracy as well as the amount of detailed information that can be gathered from their use. However, the inherent complexity and large computational requirements of these modelling schemes may not serve as the best tools to give the researcher a high level, "big picture" understanding of the fundamental physics governing hot-arm actuator behaviour. In one attempt to address this issue, Adams [16] performed a dimensional analysis on the simplified hot-arm actuator shown in Figure 2.
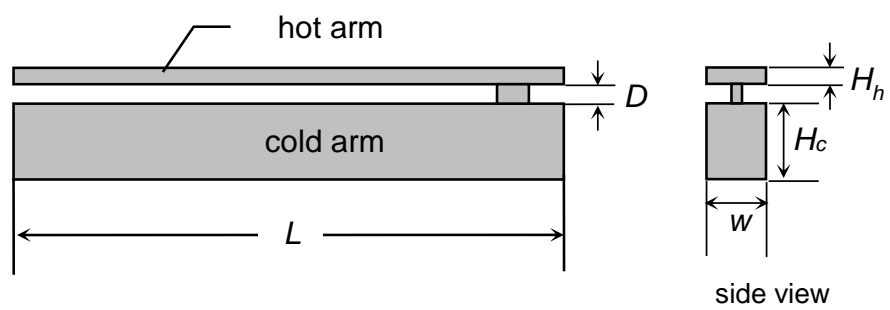

Fig. 2: Simplified hot arm actuator for which dimensional analysis is performed in [16]. The flexure has been removed in order to reduce the geometric complexity.

In [16] Adams derived two main dimensionless pi groups governing the behaviour of hot-arm actuation, the Biot number $(B i)$ and a new pi group dubbed the thermo-electro parameter, $P o$,

$$
\begin{gathered}
B i=\frac{h w}{k}, \\
P o=\frac{\alpha V^{2}}{\rho h w},
\end{gathered}
$$


where $h$ is the convective heat transfer coefficient between the actuator and the surroundings, $k$ is the thermal conductivity of the actuator, $\alpha$ is the thermal expansion coefficient, $V$ is the applied voltage, and $\rho$ is electrical resistivity. This thermoelectro parameter contains variables related to the Joule heating of the device, the surroundings' ability to dissipate that heat, and the tendency of the material to expand with increased temperature. It may be interpreted as a relative measure of the overall thermal strain of the actuator. Furthermore, the deflection in hot-arm actuation is directly proportional to the thermoelectro parameter, an observation that is consistent with many previous studies in which deflection is proportional to electrical power dissipation and the coefficient of thermal expansion $[1,2,4,8]$.

Also shown in [16] was that the Biot numbers encountered in hot-arm actuation are consistently on the order of, or less than, 0.1. This is true even when $B i$ is based on length of the actuator $L$ instead of thickness $w$. This calls into question the need for detailed knowledge of temperature distribution in modelling hot-arm actuators, at least as far as predicting deflection is concerned. Lastly, it was shown that there is no way to account for the dimensions of Young's modulus, $E$, in creating the dimensionless pi groups. The suspicions voiced in [4] and [11] that hot-arm deflection is insensitive to Young's modulus were thus proven.

Left out of the analysis of [16], however, was the role of the additional dimensionless pi terms that result from the physical lengths shown in Fig. 2. This is in addition to modelling the actuator as having no flexure. Clearly, the geometry of the hot-arm actuator plays a major role in its operation, and is therefore worthy of closer study.

\section{Dimensional Analysis}

The goal of the present work is to elaborate on the work of [16] by including the effect of geometry via additional dimensionless group(s). Specifically, we seek to correlate existing data in order to better understand the effect of geometry on hot-arm actuation, and to produce a dimensionless correlation that predicts deflection for any hot-arm actuator, regardless of its dimensions and material construction.

\subsection{Dimensionless Groups}

Figure 3 gives a schematic diagram of a traditional, U-shaped hot-arm actuator, along with the notation used for the various dimensions.
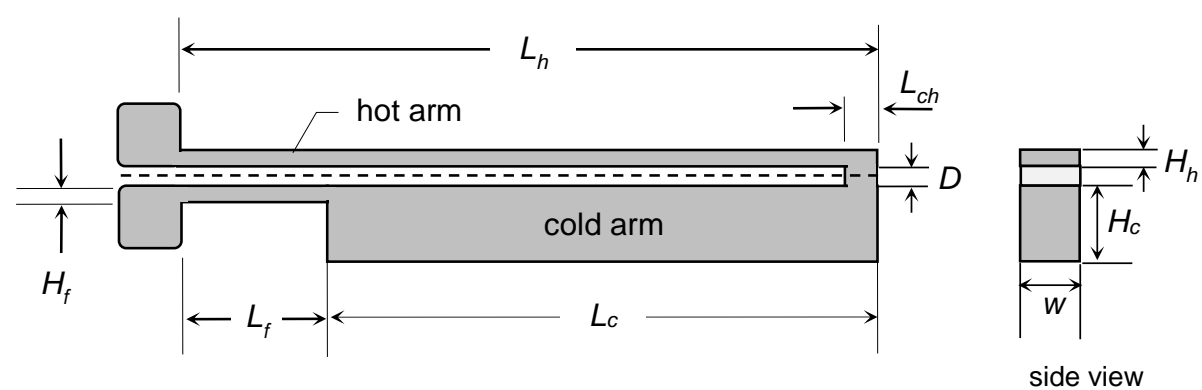

Fig. 3: Notation used for dimensions of the hot-arm actuator. The dotted line shows the assumed neutral axis, and is halfway between the bottom of the hot arm and the top of the cold arm/flexure.

The tip deflection of the actuator (see Fig. 1) is a function of the dimensions shown in Fig. 3 along with the other physical parameters already discussed:

$$
\omega=f\left(w, L_{h}, L_{f}, L_{c}, L_{c h}, H_{f}, H_{h}, H_{c}, D, h, E, V, \rho, \alpha, k\right) \text {. }
$$

The total number of variables in the relation given in Eq. (3) is $s=16$, requiring $r=5$ reference dimensions (length, energy, charge, time, and temperature). The Buckingham Pi theorem allows the functional form of Eq. (3) to be reduced to a relationship among $n=s-r=11$ dimensionless pi groups. The derivation of the pi terms parallels that in [16] and is not reproduced here. The result is

$$
\omega / w=f\left(B i, P o, \Pi_{\mathrm{n}}\right)
$$


where $\omega / w$ is the dimensionless tip deflection; $B i$ and $P o$ are the Biot number and thermo-electro parameter as given by Eqs. (1) and (2), respectively; and $\Pi_{\mathrm{n}}$ represents eight dimensionless groups each having the form $L_{x} / w, L_{x}$ being one of the eight lengths shown in Fig. 3 excluding the actuator thickness, $w$.

Seeking a functional dependence of actuator deflection on each of eight different dimensionless lengths is not practical, nor is it liable to be fruitful. It is more likely that the deflection's dependence on geometry is described by a smaller number of pi groups formed by some combination of the various lengths given in Fig. 3. Furthermore, [16] suggests that the actuator deflection dependence on $B i$ is negligible. For simplicity, a correlation having the following form is therefore sought:

$$
\omega / w=f(P o, G o)
$$

where Go is a geometric dimensionless group formed from a yet to be determined combination of the lengths shown in Fig. 3 .

In seeking a candidate for Go, we first refer to Adams and Layton [4], in which an elementary, lumped-element model for the simplified hot-arm actuator shown in Fig. 2 is presented. The model produces a single, closed form equation predicting hot-arm deflection as a function of applied voltage. Created as a pedagogical tool, the model incorporates many simplifications - most notably the absence of a flexure - that severely limit its predictive accuracy. Germane to the current work, however, is the dependence of the deflection on the combination of dimensions given by $D A_{h} w / I_{c}$, where $A_{h}$ is the cross sectional area of the hot arm and $I_{c}$ is the second moment of area of the cold arm.

One possibility for $G o$ would be to replace $I_{c}$ with a more appropriate quantity representing the average second moment of inertia for an entire traditional hot-arm actuator including a flexure. Seng et al [15] employ such scheme in calculating a linearly weighted height of the actuator. For the actuator shown in Fig. 3, this is

$$
H_{\text {avg }}=\frac{L_{f}}{L_{h}}\left(H_{f}+H_{h}\right)+\frac{L_{c}-L_{c h}}{L_{h}}\left(H_{c}+H_{h}\right)+\frac{L_{c h}}{L_{h}}\left(H_{h}+D+H_{c}\right)
$$

The average height is then used to approximate the second moment of inertia as $I_{\text {avg }}=w H_{\text {avg }}{ }^{3} / 12$. As an alternative, the present work calculates a linearly weighted average of the second moment of area itself,

$$
I_{\text {avg }}=\frac{L_{f}}{L_{h}} I_{f+h}+\frac{L_{c}-L_{c h}}{L_{h}} I_{c+h}+\frac{L_{c h}}{L_{h}} I_{c h}
$$

where each moment of area is calculated for the appropriate section of the actuator, and then corrected to be about the axis halfway between the bottom of the hot-arm and the top of the cold-arm/flexure. Using this average second moment of area, the geometric parameter becomes

$$
G o=\frac{D A_{h} w}{I_{a v g}}=\frac{D H_{h} w^{2}}{I_{a v g}} .
$$

The gap distance between the hot and cold arms, $D$, arises in Eq. (8) from approximating the internal bending moment produced in the actuator upon heating. In general, the compressive stress in the hot arm produces greater moments for larger values of gap distance, $D$. The denominator of Eq. (8) represents the stiffness of the actuator as a whole. Thus, Go represents the ratio of the induced bending moment to the actuator's resistance to that bending; in short, a measure of the geometric "bendability" of the actuator.

\subsection{Reduced Data and Discussion}

Data for actuator deflections were reduced for six data sets retrieved from five different studies ([5], [8], and [12-14]) using the assumed functional form of Eq. (5) and the dimensionless groups Po and Go. Data were taken and/or calculated from the references themselves. 
Figure 4 shows the dimensionless actuator deflection as a function of the thermo-electro parameter, Po. Also shown are least-squares linear fits of the data. For all six sets of data the correlation coefficient is greater than 0.98 , showing a highly linear dependence between deflection and Po. This linear behaviour agrees with numerous previous investigations that have shown a direct dependence of deflection on electrical power dissipation.

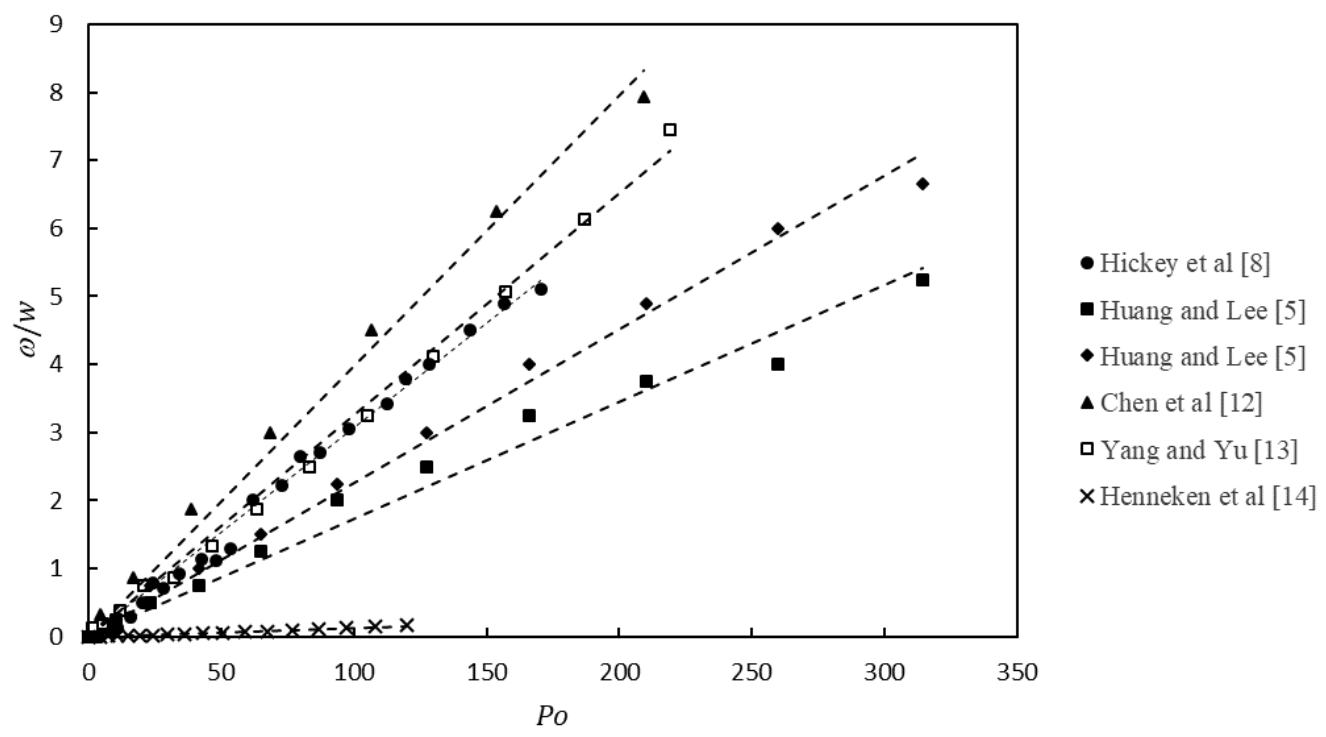

Fig. 4: Variation of dimensionless actuator deflection with Po for six different actuators.

Figure 5 explores the role of actuator geometry in the deflection-Po relationship. In Fig. 5 the slopes of each best-fit line in Fig. 4 are plotted as a function of the geometric parameter, Go. A power law dependence with a slope of $m=0.002 G o^{-0.64}$ gives the best fit of the data. The dimensionless deflection of Eq. (5) thus becomes

$$
\omega / w=0.0020 P o G o^{-0.64}
$$

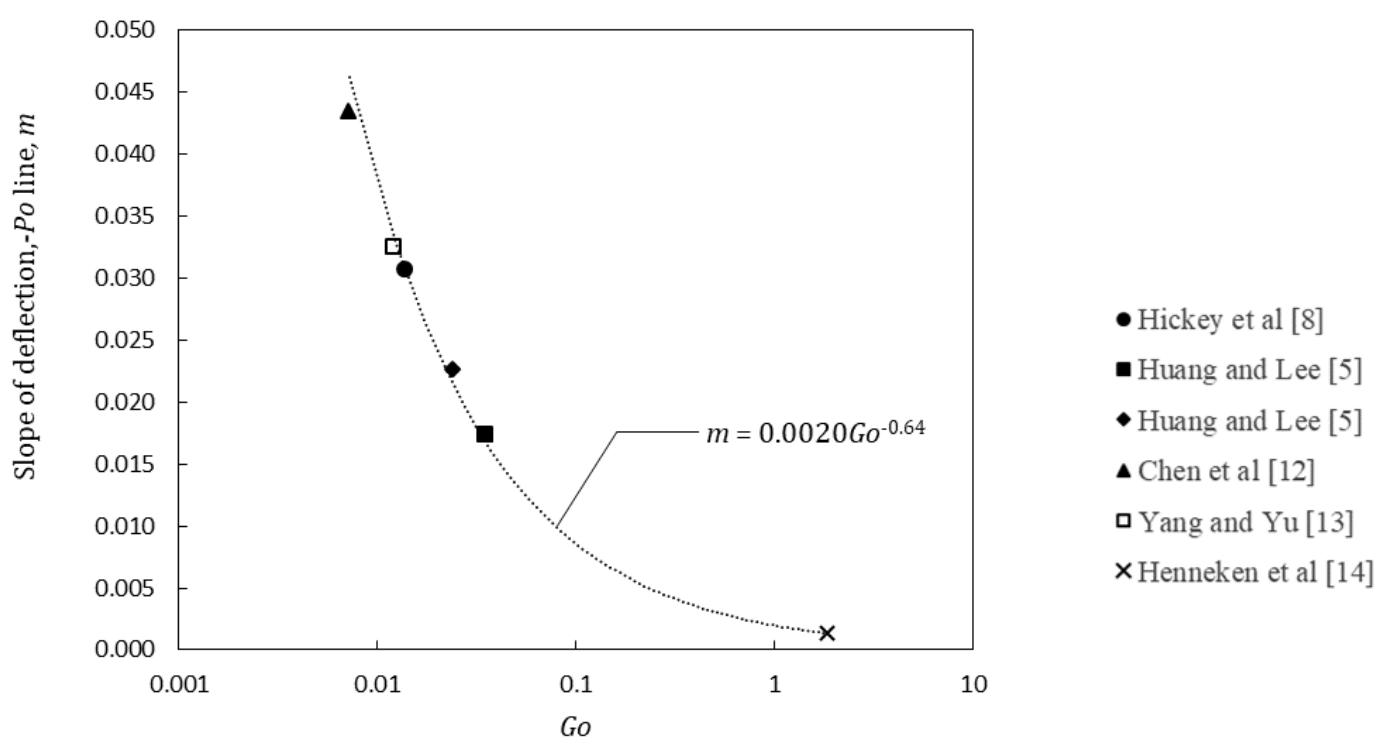

Fig. 5: Deflection-Po slope dependence on the geometric parameter, Go. 
At first inspection the decreasing deflections realized at higher values of Go in Fig. 5 may seem counterintuitive. Higher values of $I_{\text {avg }}$ represent stiffer structures, which should be more difficult to bend. As a larger $I_{\text {avg }}$ results in a lower value of Go, one might expect less bending at small Go, and therefore smaller deflections as well. However, a distinction should be made between tip deflection and the bending of the entire actuator. In fact, it is the rigid body like behaviour of the cold arm-whose second moment of area dominates the value of $I_{\text {avg }}$ - that most likely accounts for the larger deflections of actuators with small values of Go. Figure 6 illustrates this idea.

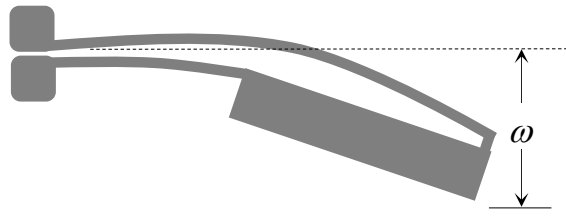

(a)

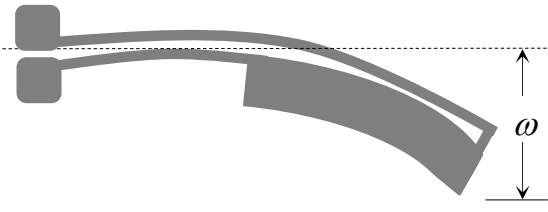

(b)

Fig. 6: Large bendability does not directly result in large actuator defection. (a) A stiff cold arm results in a large tip deflection. (b) More bending in the cold arm can result in less tip deflection.

Figure 7 compares dimensionless deflections as predicted from Eq. (9) against the experimentally measured values for all sets of data from all studies. A perfect prediction would lie on a line with a slope of unity. As can be seen from the figure, Eq. (9) does a good job predicting actuator deflection, predicted values regularly being within $10 \%$ of the measured values.

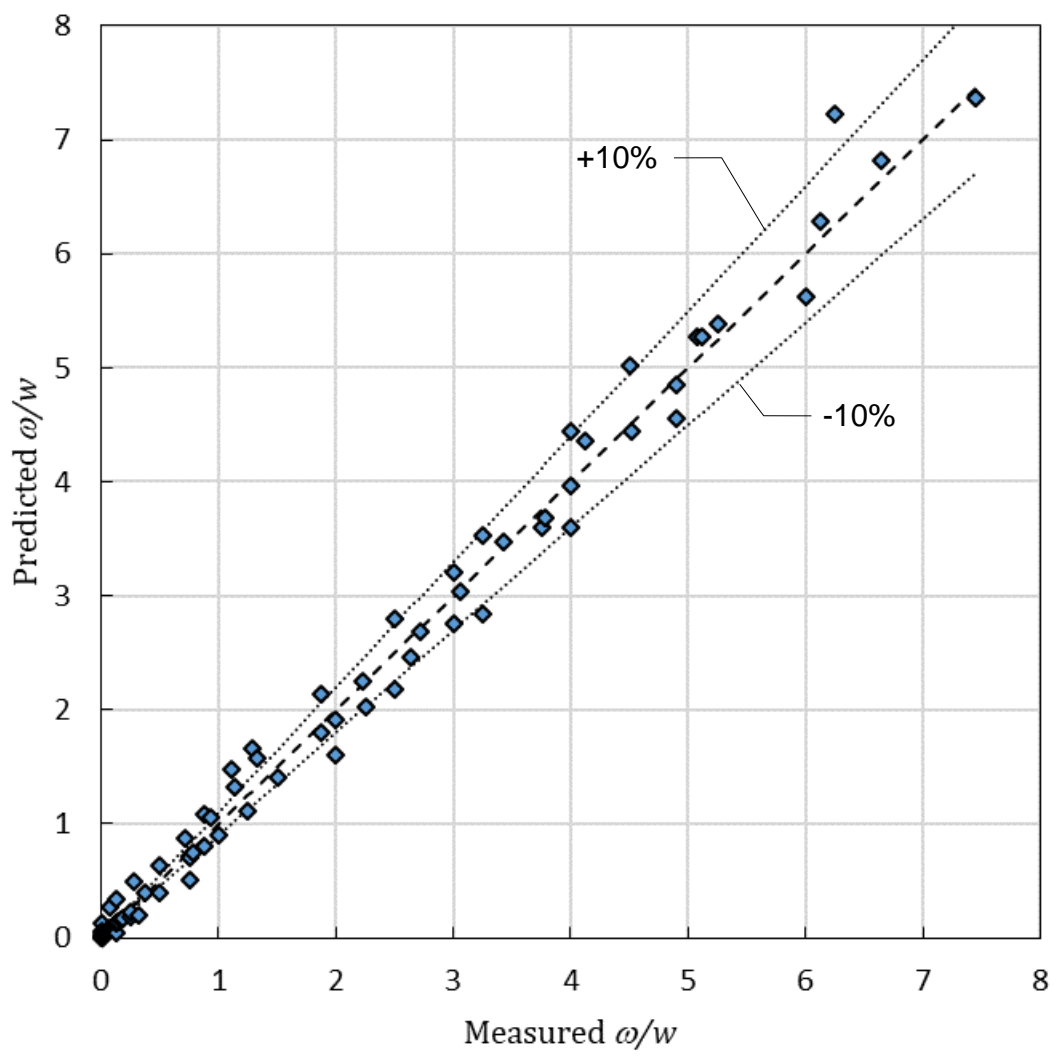

Fig. 7: Comparison of measured actuator deflections from [5], [8], and [12-14] to those predicted by Eq. (9). Predictions consistently fall within $\pm 10 \%$ of the actual measured values. 


\section{Conclusion}

The effect of dimensionless parameters on the deflection of MEMS hot-arm actuators has been studied. Deflections from six sets of data, collected from five different studies in the literature, are well correlated via two dimensionless groups, a thermo-electro parameter and a geometric parameter. Actuator deflection shows a strong linear dependence on the thermoelectro parameter, which is a rough measure of the overall thermal strain of the actuator. This linear dependence is consistent with several previous studies. Larger values of the geometric parameter, which represents the bendability of the actuator, result in smaller tip deflections. The semi-empirical relationship developed in the current study is well suited to aiding in the design and analysis of MEMS hot-arm actuators.

\section{Acknowledgements}

The authors wish to thank the Operation Catapult summer program at Rose-Hulman Institute of Technology for providing them with the opportunity to participate in this research.

\section{References}

[1] J. H. Comtois, M. A. Michalicek, and C. C. Barron, "Electrothermal actuators fabricated in four-level planarized surface micromachined polycrystalline silicon," Sensors and Actuators A, vol. 70, pp. 23-31, 1998.

[2] A. Siahmakoun, S. Kirkpatrick and Z. Roth, "Array of MEMS Mirrors for Beamforming," ASNE 2003 Symposium, Bloomington, IN, Sep. 6-8, 2003.

[3] J. H. Comtois and V. M. Bright, "Applications for surface-micromachined polysilicon thermal actuators and arrays," Sensors and Actuators A, vol. 58, pp. 19-25, 1997.

[4] T. M. Adams and R. A. Layton, Introductory MEMS: Fabrication and Applications. New York, NY: Springer, 2010.

[5] Q. A. Huang and N. K. S. Lee, "Analysis and design of polysilicon thermal flexure actuator," Journal of Micromechanics and Microengineering, vol. 9, pp. 64-70, 1999.

[6] L. Li and D. Uttamchandani, "Modified asymmetric microelectrothermal actuator: analysis and experimentation," Journal of Micromechanics and Microengineering, vol. 14, pp. 1734-1741, 2004.

[7] C. D. Lott, T. W. McLain, J. Harb, and L. Howell, "Thermal modeling of a surface-micromachined linear thermomechanical actuator," in Modeling and Simulation of Microsystems, pp. 370-373, 2001.

[8] R. Hickey, M. Kujath, and T. Hubbard, "Heat transfer analysis and optimization of two-beam microelectromechanical thermal actuators," Journal of Vacuum Science \& Technology A, vol. 20, pp. 971-4, 2002.

[9] N. D. Mankame and G. K. Ananthasuresh, "Comprehensive thermal modeling and characterization of an electrothermal-compliant microactuator," Journal of Micromechanics and Microengineering, vol. 11, pp. 452-62, 2001.

[10] D. Yan, A. Khajepour and R. Mansour, "Modeling of two-hot-arm horizontal thermal actuator," Journal of Micromechanics and Microengineering, vol. 13, pp. 312-322, 2003.

[11] T. M. Adams, A. J. Bomar, A. J., and S. Kirkpatrick, "Lumped Element Model for a MEMS Hot Arm Actuator," in Proceedings of the CSME Forum, Victoria, BC, Canada 2010.

[12] R. S. Chen, C. Kung, and G.-B. Lee, "Analysis of the optimal dimension on the electrothermal microactuator," Journal of Micromechanics and Microengineering, vol. 12, pp. 291-296, 2002.

[13] Y.-J. Yang and C.-C. Yu, "Extraction of heat-transfer macromodels for MEMS devices," Journal of Micromechanics and Microengineering, vol.14, pp. 587-596, 2004.

[14] V. A. Henneken, M. Tichem, and P. M. Sarro, "Improved thermal U-beam actuators for micro-assembly," Sensors and Actuators A, vol. 142, pp. 298-305, 2008.

[15] A. B, Seng, Z. Dahari, O. S., and M. A. Miskam, "Design and Analysis of Thermal Microactuator," European Journal of Scientific Research, vol.35 no.2, pp.281-292, 2009.

[16] T. M. Adams, "Dimensional Analysis of a Hot-arm Actuator," In Proceedings of 4th International Conference on Heat Transfer and Fluid Flow, Rome, Italy, June 9-10, 2017. 